\title{
Seismic Performance of Steel and Concrete Composite Shear Walls with Embedded Steel Truss for Use in High-Rise Buildings
}

\author{
Yun-tian Wu*, Dao-yang Kang, Yeong-Bin Yang \\ School of Civil Engineering, Chongqing University, Chongqing 400045, China \\ *Corresponding author: yuntianw@cqu.edu.cn
}

This paper studies the seismic behavior of steel and concrete composite shear walls with embedded steel truss, a crucial structural element for use in high-rise buildings. Three one-fourth scaled composite wall specimens with an aspect ratio of 1.0 were tested until to failure under reversed cyclic lateral load and constant axial load. The test parameters were the amounts of embedded truss chord and web brace. The behavior of the test specimens, including the damage formation, failure mode, hysteretic curve, stiffness and strength degradation, energy dissipation and ductility, were examined. Test results indicated that the embedded truss web braces affect significantly the hysteretic behavior of the composite walls in terms of lateral load capacity, energy dissipation and ductility, while the embedded truss chords can enhance the lateral load capacity. To further broaden the test results obtained, while searching for the optimal design, finite element (FE) models were validated against all the test results. Then 27 FE models that cover the practical ranges of axial load ratio, amount of embedded steel truss chord and web braces were adopted in a parametric analysis to investigate their effects on the wall performance. The results indicate that high axial load ratio is beneficial to initial stiffness and lateral load capacity, while adverse to energy dissipation capacity. Although increasing the volumetric ratio of embedded truss web brace can most effectively increase the lateral load capacity, a medium level about $1.59 \%$ is its optimum value for ensuring the critical seismic performance in terms of energy dissipation and ductility. In order to avoid the adverse effect on ductility behavior, the axial load ratio should be limited to the medium level, about 0.13 for the nominal value or about 0.30 for the design value.

Keywords: composite wall; static test; earthquake; parametric analysis; shear wall; steel truss 


\section{Introduction}

In recent years, a large number of high-rise reinforced concrete (RC) buildings have been constructed in regions of high seismicity. If conventional RC shear walls are used as the lateral force resisting system, the thickness of wall may need to be significantly increased in order to satisfy the stringent seismic design provisions of current building codes, resulting in reduced usable floor area and increased seismic structural weight. The difficulty and cost of the construction will also be increased. Therefore, the steel and concrete composite wall has been introduced, where shaped steel is incorporated into RC wall, to obtain high strength, high stiffness and excellent post-yield deformation characteristics by combining the advantages of concrete and steel materials.

Various types of steel and concrete composite walls have been developed and used in seismic zones. Zhao and Abolhassan [1] attached RC panels to the steel plate walls using bolts, resulting in highly ductile behavior and stable cyclic post-yielding performance. Dan et al. [2] encased vertical steel profiles into RC walls and demonstrated the effectiveness of the steel profiles in improving the seismic performance of RC shear walls. Qian et al. [3] embedded steel tubes at the wall boundaries and fully anchored into the foundation of $\mathrm{RC}$ walls and proved such composite details can ensure good seismic performance under high axial load and cyclic lateral loading. Nie et al. [4] investigated the seismic behavior of high-strength composite walls composed of concrete filled steel tubular (CFT) columns at the two boundaries and high-strength concrete filled double-steel-plate wall body. Rafiei et al. 
[5] and Hossain et al. [6] investigated the behavior of composite shear walls consisting of two skins of profiled steel sheeting and an infill of concrete under in-plane monotonic and cyclic loading respectively, demonstrating more ductile behavior and higher energy absorbing capacity. Chen et al. [7] studied the double steel plate-high strength concrete composite walls with concrete filled steel tube boundary elements, showing high strength and excellent deformation capacity.

In this study, an alternative type of steel and concrete composite shear wall has been developed as shown in Fig. 1. The steel truss embedded in the concrete wall is composed of channel steel chords at boundary elements and equal-leg double angle web braces at the wall web. By embedding a steel truss instead of steel plate in the RC wall, considerable saving in steel material and improvement in constructability can be achieved. Previously, Sittipunt et al. [8] and Shaingchin et al. [9] have proved the effectiveness of diagonal web reinforcement in improving the seismic performance of the RC walls. Replacing the diagonal web reinforcing bars by shaped steel members such as equal-leg double angle can surely improve the constructability. In order to further investigate the seismic behavior of steel and concrete composite walls embedded with steel truss, a series of three one-fourth scaled wall specimens with a small aspect ratio of 1.0 were tested under constant axial load and reversed cyclic lateral loads. To broaden the results obtained, while searching for the optimal design, finite element models were established and validated via comparison of the simulation results with the experimental ones for the three specimens. Then 27 FE models that cover the practical ranges of axial load ratio, steel ratio of embedded truss chord 
members, and volumetric ratio of embedded truss web braces were adopted in the parametric study to investigate their influences on the overall performance of the composite shear walls.

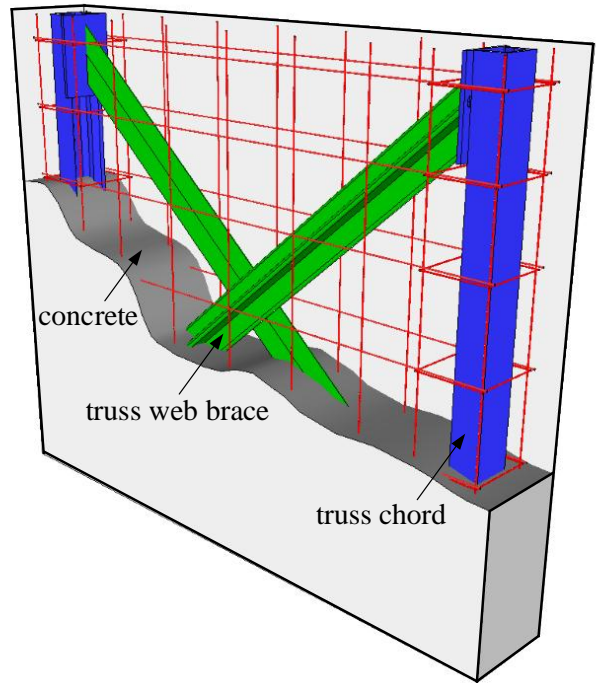

Fig. 1 Proposed type of composite wall

\section{Experimental Program}

\subsection{Details of test specimens}

Three one-fourth scaled specimens embedded with steel truss identified by S-1, S-2 and S-3 were constructed and tested under constant axial load and reversed cyclic lateral load. All the specimens were designed according to the Chinese Code for Seismic Design of Buildings (GB50011-2010) [10], having identical overall geometric dimensions, as shown in Fig. 2. The aspect ratio of the wall height $l_{\mathrm{w}}$ to wall width $h_{\mathrm{w}}$ was approximately 1.0. The chord members of the embedded truss were channel steel for specimens S-1 and S-3 and I section steel for specimen S-2. The web brace member of the embedded truss was equal-leg double steel angles $\llcorner 25 \times 2$ for specimens $S-1$ and $S-2$ and $\llcorner 30 \times 4$ for $S-3$. The shop welded $180 \mathrm{~mm} \times 68 \mathrm{~mm} \times 6 \mathrm{~mm}$ 
endplate connection was introduced to join the chord and web brace of the embedded truss. Detailed dimensions of the truss members are also shown in Fig. 2.

The main design results of the specimens are listed in Table 1. The key parameters of the specimens are listed in Table 2, where the steel ratio $\rho_{\mathrm{c}}$ of the embedded truss chord is defined as the ratio of the cross sectional area of the chord members to the total wall cross sectional area, and the volumetric steel ratio $\rho_{\mathrm{vb}}$ of the embedded truss web brace is defined as the ratio of the web braces volume to that of the wall. The steel ratios of the embedded truss chord members were $1.73 \%$ for S-1 and S-3 and $3.59 \%$ for specimen S-2. The volumetric steel ratios of the embedded truss web braces were $0.70 \%$ for specimens S-1 and S-2 and $1.59 \%$ for specimen S-3. The influences of $\rho_{\mathrm{c}}$ and $\rho_{\mathrm{vb}}$ on the overall behavior of the composite wall can be evaluated by the comparison of the test results of the specimens. The axial load ratio $\alpha$, defined as the ratio of the applied axial load to the total axial load carrying capacity of the wall cross section, was 0.2 for all three specimens. It was approximately maintained constant and vertical during testing.
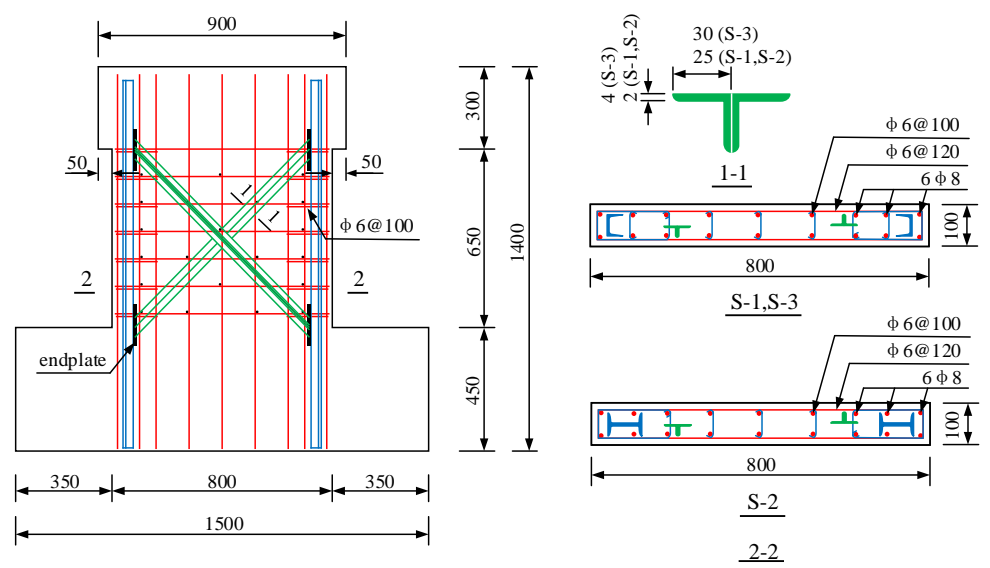

Fig. 2 Detailed dimensions of the specimens 
Table 1 Design data of specimens

\begin{tabular}{|c|c|c|c|}
\hline Specimen & S-1 & S-2 & S-3 \\
\hline Length $\times$ Thickness $\times$ Height & \multicolumn{2}{|c|}{$800 \mathrm{~mm} \times 100 \mathrm{~mm} \times 650 \mathrm{~mm}$} \\
\hline Horizontal reinforcement & $\phi 6 @ 100$ & $\phi 6 @ 100$ & $\phi 6 @ 100$ \\
\hline Vertical reinforcement & $\phi 6 @ 120$ & $\phi 6 @ 120$ & $\phi 6 @ 120$ \\
\hline Boundary reinforcement & $6 \phi 8$ & $6 \phi 8$ & $6 \phi 8$ \\
\hline Embedded truss chord & $\mathrm{C} 5$ & $\mathrm{I} 10$ & $\mathrm{C} 5$ \\
\hline Cross sectional area of chord $\left(\mathrm{mm}^{2}\right)$ & 692 & 1435 & 692 \\
\hline Embedded truss web brace & $2\llcorner 25 \times 2$ & $2\llcorner 25 \times 2$ & $2\llcorner 30 \times 4$ \\
\hline Cross sectional area of brace $\left(\mathrm{mm}^{2}\right)$ & 192 & 192 & 456 \\
\hline Axial compression load $(\mathrm{kN})$ & 837 & 883 & 827 \\
\hline
\end{tabular}

Table 2 Key parameters of specimens (\%)

\begin{tabular}{|c|c|c|c|}
\hline Specimen & S-1 & S-2 & S-3 \\
\hline$\rho_{\mathrm{c}}$ & 1.73 & 3.59 & 1.73 \\
\hline$\rho_{\mathrm{vb}}$ & 0.70 & 0.70 & 1.59 \\
\hline
\end{tabular}

\subsection{Test Setup and Instrumentation}

The axial load and lateral cyclic load were applied to the specimens using the test setup shown in Fig. 3. It is noted that there was a roller track between the vertical hydraulic jack and the reaction girder that allowed the jack to horizontally slide so as to maintain the vertical direction of the axial load. The reversed cyclic horizontal load was applied to the specimen by a servo-controlled hydraulic actuator attached horizontally to the reaction wall with the maximum load capacity of $1000 \mathrm{kN}$ and a stroke of $\pm 250 \mathrm{~mm}$. For convenience, the pushing and pulling of the horizontal actuator were regarded as the positive and negative loading directions, respectively. 


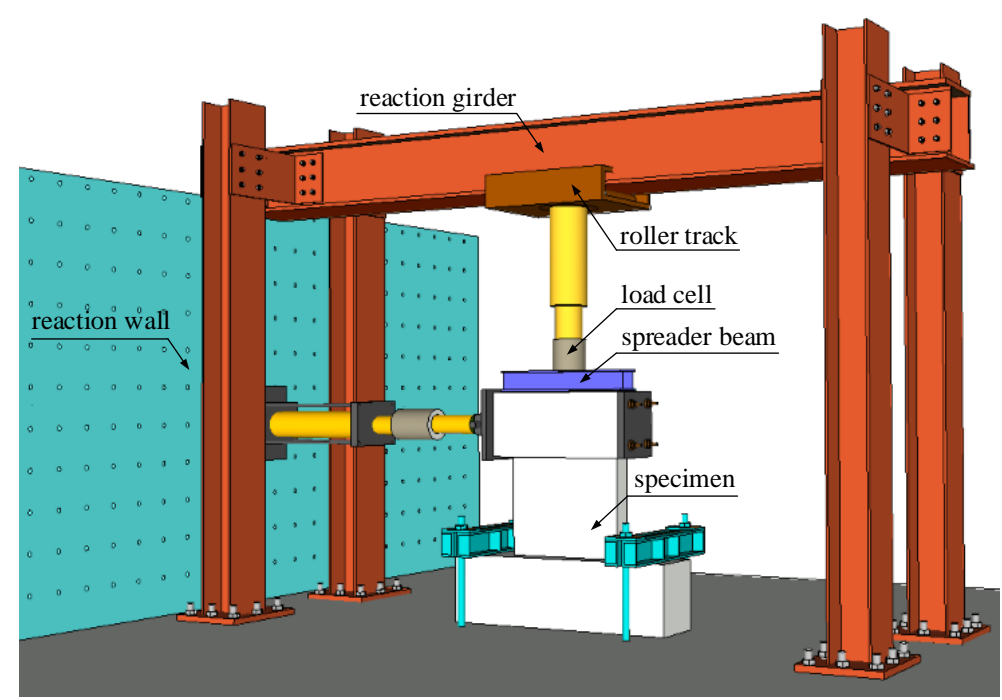

Fig. 3 Test setup

The lateral cyclic loading procedure was divided into the load-controlled and displacement-controlled stages. The load-controlled stage started from loading in the positive direction until the first cracking was observed. Then similar loading pattern was applied in the negative loading direction until the cracking was found. Subsequently, the loading cycles were displacement controlled by a displacement increment of $1.5 \mathrm{~mm}$. When the specimen started to yield, which was determined according to the strain reading of the outermost longitudinal reinforcing steel at bottom wall section, a displacement increment of $3 \mathrm{~mm}$ was used till the test was terminated. Two cycles of loading were repeated corresponding to each displacement level.

The main instrumentation plan is shown in Fig. 4. Displacement transducers were mounted against a rigid reference frame to measure the lateral displacement at 300 mm intervals over the wall height. A pair of diagonally placed displacement transducers were installed on the wall surface of each specimen to measure the shear deformation. Linear potentiometers with a stroke of $\pm 20 \mathrm{~mm}$ were used to measure 
the flexural deformation of the wall base. Strain gages were attached to critical locations of reinforcing bars (not shown in Fig. 4) and embedded steel truss members to monitor the deformation.

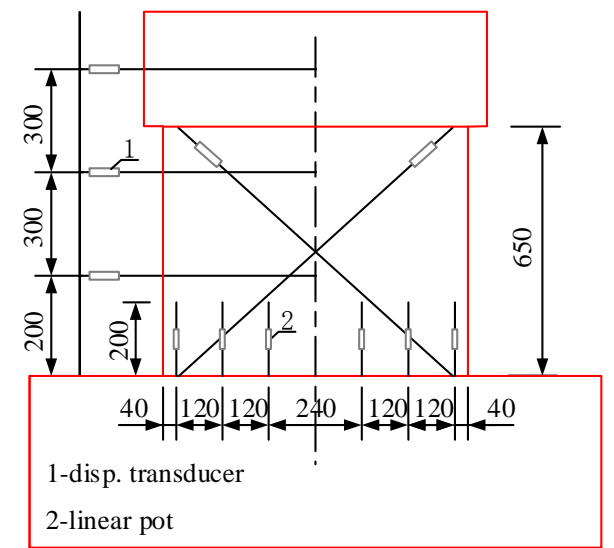

a) Displ. transducer and linear pot

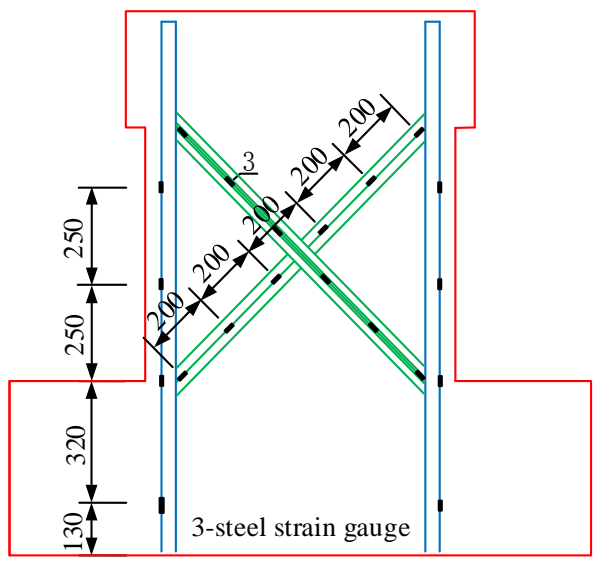

b) Strain gauges on shape steel

Fig. 4 Instrumentation

\subsection{Material Properties}

High strength concrete with nominal compressive strength of $70 \mathrm{MPa}$ was used for all three specimens. The average compressive strength of $150 \mathrm{~mm} \times 150 \mathrm{~mm} \times 150 \mathrm{~mm}$ standard cubic samples measured on the $28^{\text {th }}$ curing day were $69.3,69.3$ and $68.5 \mathrm{MPa}$ for the three specimens, respectively. The main mechanical properties of the reinforcing bars and embedded truss steel are listed in Table 3.

Table 3 Steel material properties

\begin{tabular}{|c|c|c|c|}
\hline Shape steel type & $\begin{array}{c}\text { Yield stress } \\
f_{\mathrm{y}}\left(\mathrm{N} / \mathrm{mm}^{2}\right)\end{array}$ & $\begin{array}{c}\text { Ultimate stress } \\
f_{\mathrm{u}}\left(\mathrm{N} / \mathrm{mm}^{2}\right)\end{array}$ & $\begin{array}{c}\text { Yield strain } \\
\varepsilon_{\mathrm{y}}\end{array}$ \\
\hline $\mathrm{C} 5$ & 458.87 & 589.51 & $2.185 \times 10^{-3}$ \\
\hline $\mathrm{I} 10$ & 313.01 & 420.65 & $1.491 \times 10^{-3}$ \\
\hline$\llcorner 25 \times 2$ & 518.83 & 740.93 & $2.471 \times 10^{-3}$ \\
\hline$\llcorner 30 \times 4$ & 612.79 & 810.51 & $2.918 \times 10^{-3}$ \\
\hline$\phi 6 \mathrm{HRB}$ & 341.67 & 498.30 & $1.627 \times 10^{-3}$ \\
\hline$\phi 8 \mathrm{HRB}$ & 301.67 & 460.00 & $1.437 \times 10^{-3}$ \\
\hline
\end{tabular}




\section{Experimental Results and Discussion}

\subsection{Damage development and failure modes}

Figure 5 shows the cracking development of each specimen corresponding to the early loading stage, peak load and failure respectively. For specimen S-1, the first cracking loads $P_{\text {cr }}$ were $180 \mathrm{kN}$ and $-160 \mathrm{kN}$ in the positive and negative directions. The cracks were firstly observed at boundary elements and then extended towards the web region (Fig. 5a). Then the outermost longitudinal reinforcing bars of the boundary elements yielded at the lateral displacement of $2.7 \mathrm{~mm}$. As the lateral displacement was increased, more inclined cracks were developed along the height of the wall boundary elements, and propagated toward the bottom web region with angles of roughly 45 degrees. The chord member and web brace of the embedded truss started to yield before the lateral displacement of $5.7 \mathrm{~mm}$, since the corresponding strains were $3.025 \times 10^{-3}$ and $2.998 \times 10^{-3}$ respectively. The diagonal cracks became more and more concentrated at the web region (Fig. 5b). At the end of testing, the wall panel was almost separated into two parts by the diagonal cracks. Severe concrete spalling off on the web region was observed (Fig. 5c). The distributed reinforcements were exposed and buckled at failure (Fig. 6a).

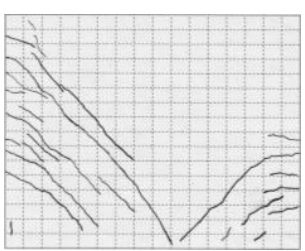

(a) S-1 at early loading stage

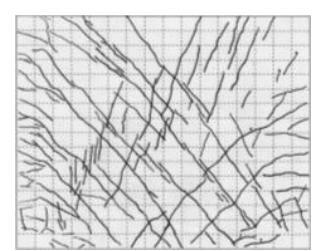

(b) S-1 at peak load

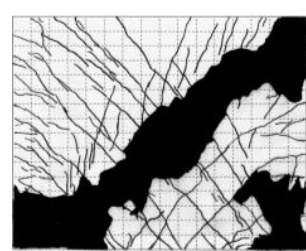

(c) S-1 at failure 


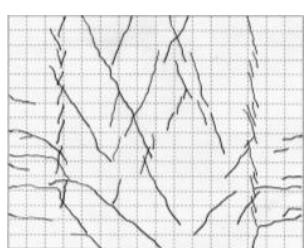

(d) S-2 at early loading stage

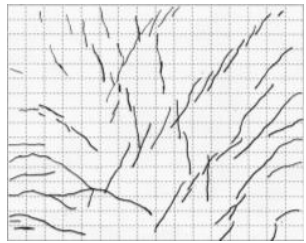

(g) S-3 at early loading stage

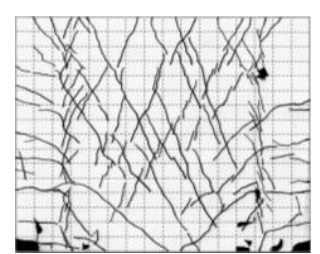

(e) S-2 at peak load

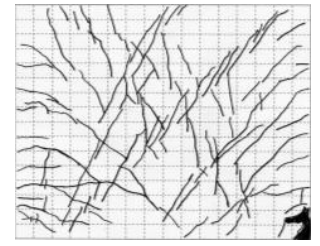

(h) S-3 at peak load

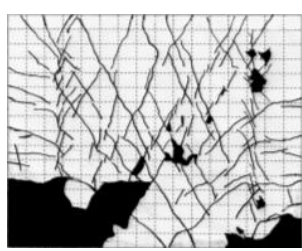

(f) S-2 at failure

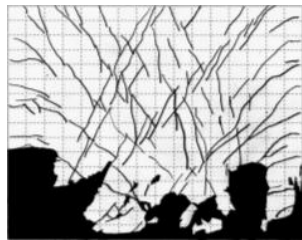

(i) S-3 at failure

Fig. 5 Cracking development of specimens

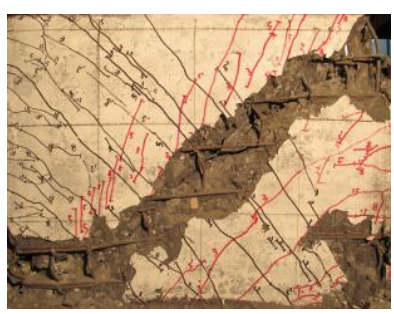

(a) $\mathrm{S}-1$

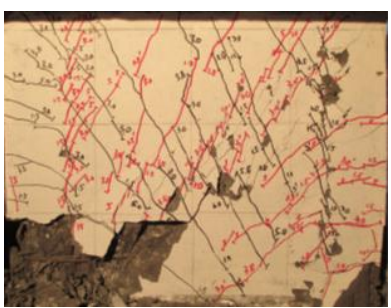

(b) $\mathrm{S}-2$

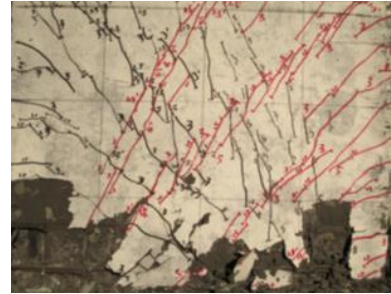

(c) $\mathrm{S}-3$

Fig. 6 Failure patterns of specimens

The cracking development processes of the three specimens were similar. The inclined cracks of specimens S-2 and S-3 approximately formed 60 and 45 degrees respectively relative to the horizontal direction. The outermost longitudinal reinforcing bars at the wall boundary on the tension sides of S-2 and S-3 yielded at the lateral displacements of 2.4 and $3.6 \mathrm{~mm}$ corresponding to tensile strains of $1.502 \times$ $10^{-3}$ and $1.489 \times 10^{-3}$ respectively. The yielding of the chord members of embedded truss of specimens S-2 and S-3 occurred prior to the lateral displacements of 5.4 and $6.6 \mathrm{~mm}$ corresponding to tensile strains of $1.608 \times 10^{-3}$ and $3.863 \times 10^{-3}$ respectively. The web braces of embedded truss of S-2 and S-3 yielded immediately after the yielding of chord members. The average cracking loads of specimen S-2 and S-3 were $230 \mathrm{kN}$ and $195 \mathrm{kN}$ respectively, which were $35.3 \%$ and $14.7 \%$ greater than that of 
specimen S-1. The spalling off and crushing of concrete at the bottom region of specimen S-3 occurred the latest among the three specimens, while specimen S-2 occurred the earliest, indicating the different post-yield deformation capacities among specimens. At the termination of testing, the web concrete crushing of S-1 appeared to be the worst as compared with specimens S-2 and S-3 (Fig. 6).

Table 4 Main test results

\begin{tabular}{|c|c|c|c|c|c|}
\hline \multirow{2}{*}{ ID } & \multirow{2}{*}{ Loading direction } & \multicolumn{2}{|c|}{ Cracking load $P_{\mathrm{cr}} / \mathrm{kN}$} & \multicolumn{2}{|c|}{ Peak load $P_{\mathrm{m}} / \mathrm{kN}$} \\
\hline & & & Average & & Average \\
\hline \multirow{2}{*}{ S-1 } & $(+)$ & 180 & \multirow{2}{*}{170} & 636 & \multirow{2}{*}{652} \\
\hline & $(-)$ & 160 & & 667 & \\
\hline \multirow{2}{*}{$S-2$} & $(+)$ & 220 & \multirow{2}{*}{230} & 716 & \multirow{2}{*}{701} \\
\hline & $(-)$ & 240 & & 685 & \\
\hline \multirow{2}{*}{ S-3 } & $(+)$ & 180 & \multirow{2}{*}{195} & 667 & \multirow{2}{*}{680} \\
\hline & $(-)$ & 210 & & 693 & \\
\hline
\end{tabular}

\subsection{Lateral Load-Displacement Relationship}

Figure 7 shows the lateral load versus displacement curves of the three specimens. It is noted that all the three specimens had a small aspect ratio of about 1.0 , resulting in the shear dominant response characteristics. The contribution of the embedded steel truss to improving the seismic behavior of the specimens varied among the three specimens. The steel ratio of the embedded truss chord for S-2 was approximately twice that of S-1. The lateral load capacities of S-2 were 716 and $685 \mathrm{kN}$ in the positive and negative directions, which were about $8 \%$ higher than those of specimen S-1, respectively. With the difference between S-1 and S-3 in the volumetric ratio of the embedded truss web braces, the lateral load capacities of specimen S-3 were 667 and $693 \mathrm{kN}$ in the positive and negative directions, which were only approximately $5 \%$ 
higher than those of specimen S-1. Due to the quick strength deterioration and shear failure after yielding, the hysteretic lateral load-displacement loops of specimens S-1 and S-2 were narrow, implying poor energy dissipation capacity. Specimen S-3 exhibited better post-yield performance than the other two specimens with much fuller hysteretic loops. The similarity of the overall characteristics of hysteretic loops between specimens S-1 and S-2 showed that the amount of the embedded truss chord had little effect on the hysteretic behavior. However, the fullest hysteretic loops of specimen S-3 among the three specimens indicated the significant influence of the embedded truss web braces on the hysteretic behavior of the composite walls under cyclic loading. The ideal lateral load carrying capacity $V_{\mathrm{n}}$, as shown in Fig. 7 by dashed lines, was calculated as the summation of $V_{\mathrm{RC}}$ and $V_{\text {st }}$, where $V_{\mathrm{RC}}$ and $V_{\text {st }}$ are the strength contributions of RC wall and embedded steel truss respectively.

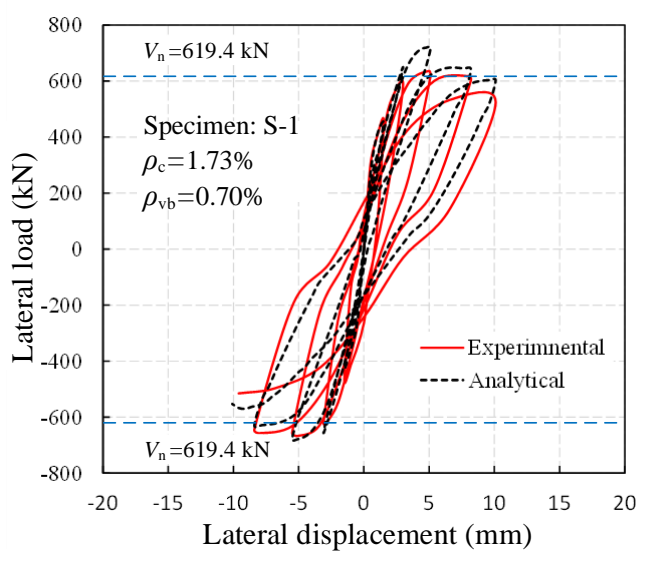

(a) Specimen S-1

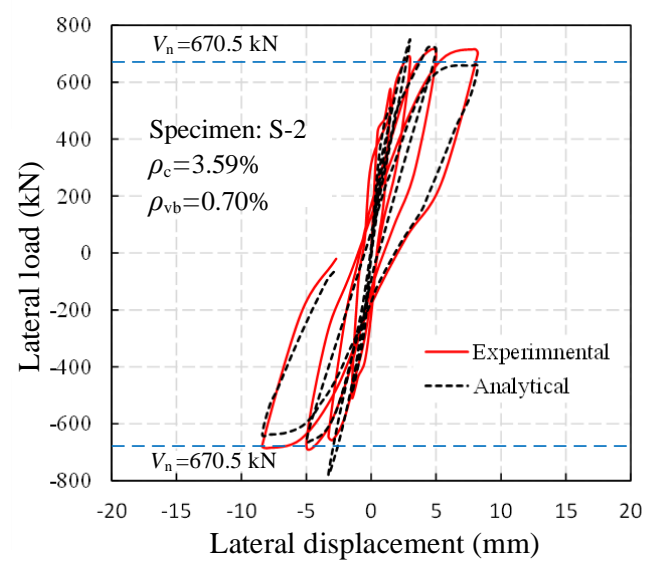

(b) Specimen S-2 


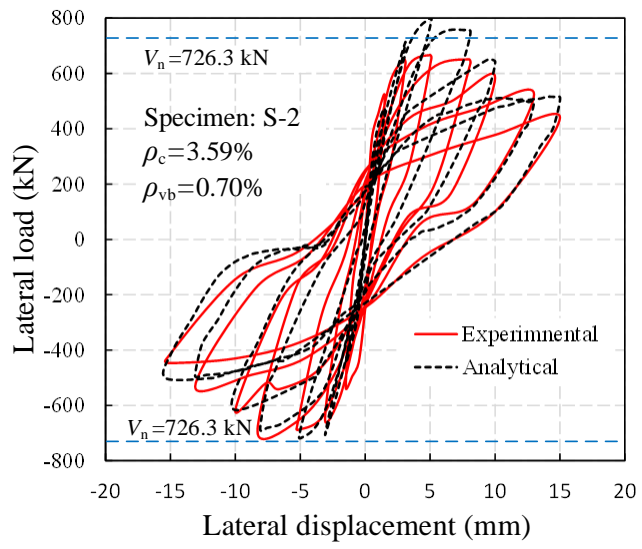

(c) Specimen S-3

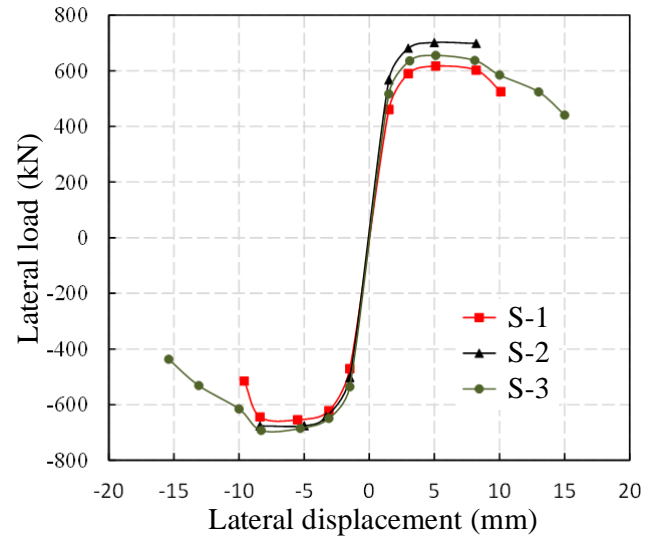

(d) Skeleton curves

Fig. 7 Lateral load-displacement responses of specimens

\subsection{Stiffness Degradation}

The stiffness degradation characteristics of the three specimens were evaluated by the secant stiffness corresponding to the lateral peak load during the first cycle of loading at each level. As shown in Fig. 8, the process of stiffness degradation is almost the same for all the specimens, indicating that the amount of the embedded steel truss has little effect on the stiffness degradation of the composite walls. The lateral stiffness of the three specimens degraded quickly in the early stages of loading due to the development of concrete cracking.

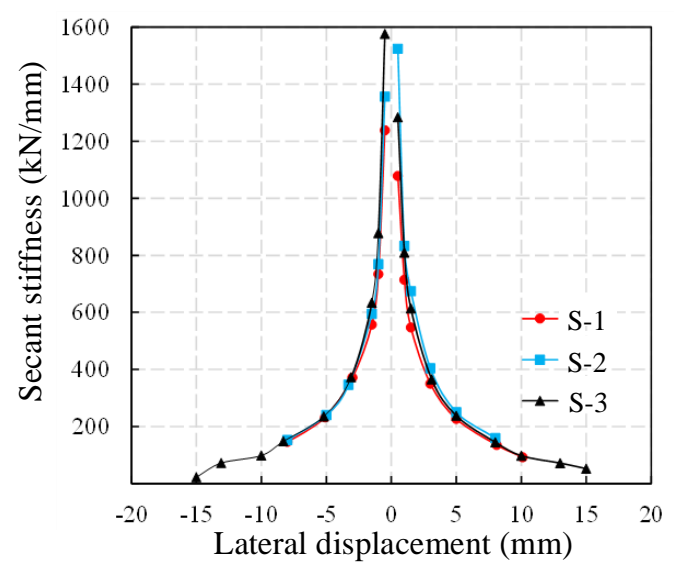

Fig. 8 Stiffness degradation of specimens 


\subsection{Energy Dissipation Capacity}

The total dissipated energy of the three specimens evaluated by the areas of the horizontal load versus lateral displacement hysteretic loops were $15.85 \mathrm{~kJ}, 18.62 \mathrm{~kJ}$ and $25.34 \mathrm{~kJ}$ respectively. Clearly, the energy dissipation capacities of S-2 and S-3 are higher than that of S-1 by 18 and $60 \%$, respectively, showing that the amount of embedded truss web braces can effectively increase the energy dissipation capacity of shear walls with small aspect ratios as compared with the embedded truss chord. Since the energy dissipation capacity plays a more important role in ensuring the structural safety of shear walls with small aspect ratios under earthquakes due to the shear dominant behavior, it is more beneficial to ensure an adequate amount of embedded truss web braces than chord members from the perspective of seismic design.

\subsection{Displacement ductility coefficient}

In order to estimate the displacement ductility of each specimen, the equivalent energy method proposed by Park [11] was adopted to determine the yield and maximum displacements based on the load-displacement skeleton curve of each specimen. The horizontal loads and displacements corresponding to initial cracking, yielding, peak lateral load and failure of the specimens in testing are listed in Table 5. The ductility of the specimens was evaluated by the displacement ductility coefficient $\mu$ calculated as $\mu=\Delta_{\mathrm{u}} / \Delta_{\mathrm{y}}$, where $\Delta_{\mathrm{y}}$ is the lateral displacement at yielding, and $\Delta_{\mathrm{u}}$ is the horizontal displacement corresponding to failure of the specimen. The displacement 
ductility coefficient $\mu$ calculated for each specimen is shown in Table 5. Evidently, the displacement ductility coefficients of S-2 and S-3 are higher than that of S-1 by about 14 and $30 \%$, respectively, indicating that the amount of embedded truss web braces has more significant influence on the displacement ductility in comparison with the embedded truss chord.

Table 5 Summary of ductility parameters for all wall specimens

\begin{tabular}{|c|c|c|c|c|}
\hline \multirow{2}{*}{ Specimen } & $\begin{array}{c}\text { Loading } \\
\text { direction }\end{array}$ & $\begin{array}{c}\text { Yield disp. } \\
\Delta_{\mathrm{y}} / \mathrm{mm}\end{array}$ & $\begin{array}{c}\text { Max. disp. } \\
\Delta_{\mathrm{u}} / \mathrm{mm}\end{array}$ & $\begin{array}{c}\text { Disp. ductility } \\
\mu\end{array}$ \\
\hline \multirow{2}{*}{ S-1 } & $(+)$ & 1.36 & 7.76 & \multirow{2}{*}{5.21} \\
\cline { 2 - 4 } & $(-)$ & 1.84 & 8.68 & \multirow{2}{*}{6.00} \\
\hline \multirow{2}{*}{ S-2 } & $(+)$ & 1.24 & - & \multirow{2}{*}{7.75} \\
\cline { 2 - 4 } & $(-)$ & 1.57 & 9.48 & \\
\cline { 2 - 5 } S-3 & $(+)$ & 1.03 & 7.66 & 9.30 \\
\hline
\end{tabular}

\section{Numerical Analysis}

To broaden the test results obtained, while searching for an optimal design, we should have recourse to the finite element (FE) analysis for a more comprehensive coverage. A parametric study based on the $\mathrm{FE}$ analysis that considers the practical ranges of all the parameters of interest, such as the axial load ratio, embedded truss chord and web braces, is a cost-effective means to understand the behavior of the composite walls. However, the FE models used should first be validated against the test results for the three specimens. This is what will be undertaken in this section.

In this paper, the proved-reliable FE analysis software DIANA [12] was used as the tool for the parametric analysis. The parameters considered include the axial load ratio $\alpha$, steel ratio of embedded truss chord $\rho_{\mathrm{c}}$, and volumetric ratio of embedded truss 
web braces $\rho_{\mathrm{vb}}$. Firstly, the finite element models for mimicking the three specimens tested were developed. The simulated results were then compared with the experimental ones to validate the accuracy of the FE modeling technique, including all the material properties and failure criteria used. Then a total of $27 \mathrm{FE}$ models that cover the practical ranges of the parameters $\alpha, \rho_{\mathrm{c}}$ and $\rho_{\mathrm{vb}}$ of interest were established and analyzed. From the comprehensive numerical simulations, the optimal design can be achieved.

\subsection{Modeling of concrete}

Three dimensional solid elements were adopted to simulate the concrete material. The total strain rotating crack model was used to simulate the cracking characteristics of concrete, in the sense that when the principal tensile stress exceeds the specified limit, cracks will occur in the perpendicular direction. The compressive stress-strain relationship of concrete used in the analysis is shown in Fig. 9. The parabolic compressive stress-strain relationship of concrete is defined by

$$
\sigma= \begin{cases}-f_{\mathrm{c}} \frac{\varepsilon_{\mathrm{j}}}{3 \varepsilon_{\mathrm{c} / 3}} & \varepsilon_{\mathrm{c} / 3}<\varepsilon_{\mathrm{j}} \leq 0 \\ -f_{\mathrm{c}} \frac{1}{3}\left(1+4\left(\frac{\varepsilon_{\mathrm{j}}-\varepsilon_{\mathrm{c} / 3}}{\varepsilon_{\mathrm{c}}-\varepsilon_{\mathrm{c} / 3}}\right)-2\left(\frac{\varepsilon_{\mathrm{j}}-\varepsilon_{c / 3}}{\varepsilon_{\mathrm{c}}-\varepsilon_{\mathrm{c} / 3}}\right)^{2}\right) & \varepsilon_{\mathrm{c}}<\varepsilon_{\mathrm{j}} \leq \varepsilon_{\mathrm{c} / 3} \\ -f_{\mathrm{c}}\left(1-\left(\frac{\varepsilon_{\mathrm{j}}-\varepsilon_{\mathrm{c}}}{\varepsilon_{\mathrm{u}}-\varepsilon_{\mathrm{c}}}\right)^{2}\right) & \varepsilon_{\mathrm{u}}<\varepsilon_{\mathrm{j}} \leq \varepsilon_{\mathrm{c}}\end{cases}
$$

where $f_{\mathrm{c}}$ is the compressive strength of concrete, $\varepsilon_{\mathrm{c} / 3}, \varepsilon_{\mathrm{c}}$ and $\varepsilon_{\mathrm{u}}$ are the concrete strains corresponding to $1 / 3 f_{\mathrm{c}}, f_{\mathrm{c}}$ and the ultimate condition, as given below: 


$$
\begin{gathered}
\varepsilon_{\mathrm{c} / 3}=-\frac{f_{\mathrm{c}}}{3 E} \\
\varepsilon_{\mathrm{c}}=-\frac{5 f_{\mathrm{c}}}{3 E}=5 \varepsilon_{\mathrm{c} / 3} \\
\varepsilon_{\mathrm{u}}=\varepsilon_{\mathrm{c}}-\frac{3 G_{\mathrm{C}}}{2 h f_{\mathrm{c}}}
\end{gathered}
$$

where $E$ is the Young's modulus and $G_{\mathrm{C}}$ is the total compressive fracture energy of concrete ranging from 10 to $25 \mathrm{~N}-\mathrm{mm} / \mathrm{mm}^{2}$ according to Feenstra [13].

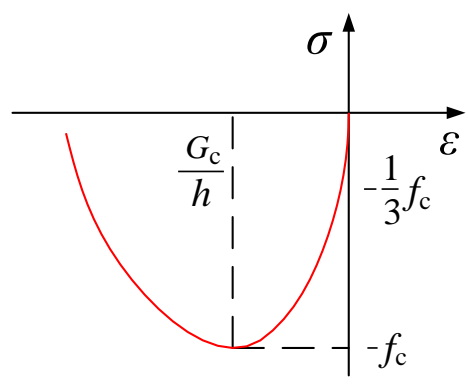

Fig. 9 Compressive stress-strain curve of concrete

The tensile stress-strain relationship of concrete used in the analysis is shown in Fig. 10. The tensile strength of concrete $f_{\mathrm{t}}$ was determined in accordance with the Chinese Code for Design of Concrete Structures (GB50010-2010) [14]. The strains $\varepsilon_{0}$ and $\varepsilon_{\mathrm{u}}$, i.e., the tensile strains corresponding to $f_{\mathrm{t}}$ and the ultimate condition, respectively, were taken as 0.000118 and 0.0014 .

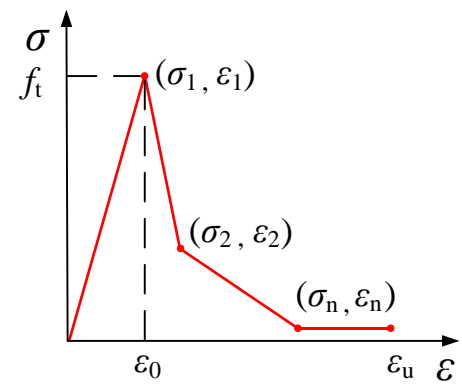

Fig. 10 Tensile stress-strain curve of concrete 


\subsection{Modeling of reinforcing bars and embedded steel truss}

The modeling of reinforcing bars and embedded steel truss members was treated differently in order to balance the simulation accuracy and computational cost. The reinforcing bars were considered using the embedded reinforcement model, where the reinforcement element is embedded in the concrete element so that the strain of the reinforcement is compatible with that of the concrete element, assuming that the reinforcement is full connection. The flat shell element, on the other hand, was used to model the embedded truss chord and web braces with the consideration of bond-slip law. The tri-linear stress-strain model, as shown in Fig. 11, was used for simulating the reinforcing bars and the shaped steel.

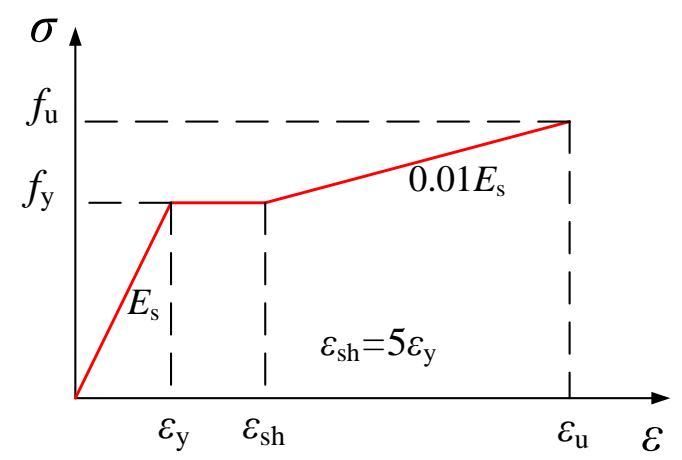

Fig. 11 Stress-strain relationship of steel

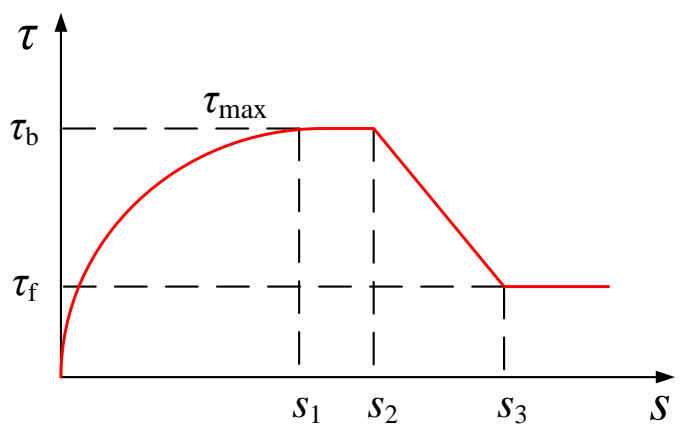

Fig. 12 Bond-slip law 


\subsection{Bond slip consideration}

The bond-slip law used in the numerical simulation is shown in Fig. 12, which is based on the CEB-FIP model code [15]. The set of equations defining the bond stress for different segments of the curve are given as follows:

$$
\begin{array}{cc}
\tau=\tau_{\text {max }}\left(\frac{s}{s_{1}}\right)^{\beta} & 0 \leq s<s_{1} \\
\tau=\tau_{\text {max }} & s_{1} \leq s<s_{2} \\
\tau=\tau_{\text {max }}-\left(\tau_{\text {max }}-\tau_{\mathrm{f}}\right) \frac{s-s_{1}}{s-s_{2}} & s_{2} \leq s<s_{3} \\
\tau=\tau_{\mathrm{f}} & s_{3} \leq s
\end{array}
$$

where $\tau$ is the bond stress, $\tau_{\max }$ is the bond strength, $\tau_{\mathrm{f}}$ is the residual bond stress, $s$ is the relative slip between steel and concrete, $s_{1}, s_{2}$ and $s_{3}$ are the characteristic slip values, and $\beta$ is an empirical coefficient. The parameters in the bond stress law depend on the steel surface condition.

\subsection{Meshing of finite element models of test specimens}

The meshing of the finite element models is important to the analysis. Although a more refined mesh can generally result in analysis results with better accuracy, the computational cost and difficulty in convergence will be increased. In the present study, the shear walls with $800 \mathrm{~mm}$ height and $650 \mathrm{~mm}$ width were equally divided into 16 and 13 segments, respectively. And the thickness of the shear wall was equally divided into two segments of $50 \mathrm{~mm}$ length. Although the element size can be further reduced to obtain a more refined mesh, no obvious difference in the calculation results was observed. A typical DIANA model of the test specimens is shown in Fig. 13. 


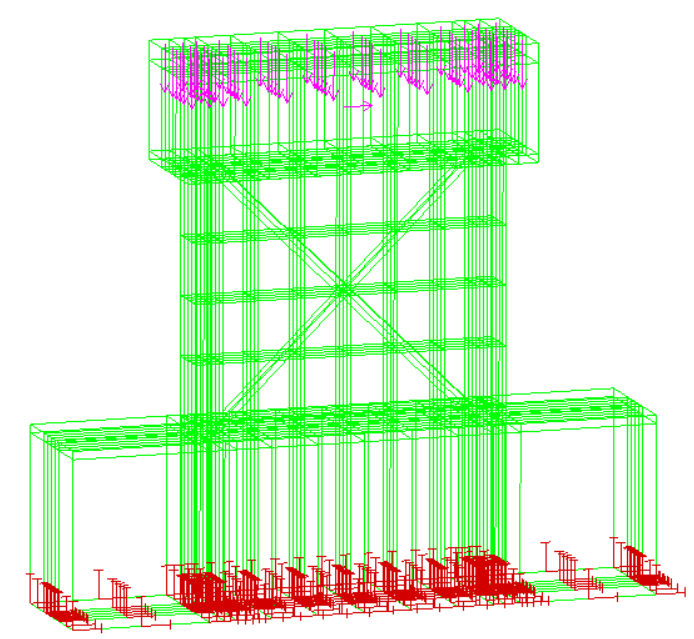

Fig. 13 Typical DIANA model of specimens

\subsection{Solution algorithm}

The regular Newton-Raphson method was adopted for solution of the nonlinear equations. Compared with the modified Newton-Raphson method, more accurate results can be obtained by the regular Newton-Raphson method. The energy convergence standard was adopted to determine if each iteration is convergent. The convergence toleration was 0.001 after the maximum iteration number of 50 times.

\subsection{Validation of Finite Element Modeling}

The effectiveness and accuracy of the finite element modeling technique was validated by comparison of the simulation results with the experimental ones. In Fig. 7, the analytical lateral load-displacement hysteretic loops (dashed lines) are plotted along with the experimental ones (solid lines). For specimen S-1 in Fig. 7(a), the simulation agrees well with the measured result in terms of loading and unloading branches of the hysteretic loops. In the positive loading direction, the simulated maximum lateral load in the vicinity of yielding point was larger than the 
experimental one. In the negative loading direction, the simulated peak load corresponding to each cycle matched very well with the measured one. For specimen S-2 in Fig. 7(b), the main deviation between the simulated and experimental results came from the abrupt increase of the simulated peak lateral load in the vicinity of yielding region in the negative direction. The loading, unloading branches of the hysteretic loops and stiffness degradation characteristics were satisfactorily captured by the numerical simulation. For specimen S-3 in Fig. 7(c), the simulated and experimental results are very close in the negative loading direction. In the positive loading direction, although the simulated peak lateral loads are higher than the experimental ones, the post-yielding behavior is well simulated. For all the three specimens the pinching effect is accurately reflected in the numerical results. In general, good agreement between the analytical and experimental results has been achieved for all the three specimens.

\section{Parametric Studies}

In the preceding section, it was confirmed that the finite element modeling is an efficient technique to capture the main mechanical characteristics of the three specimens. As a result, $27 \mathrm{FE}$ models were established to investigate the influences of axial load ratio $\alpha$, steel ratio of embedded truss chord $\rho_{\mathrm{c}}$ and volumetric ratio of embedded truss web braces $\rho_{\mathrm{vb}}$, on the seismic performance of the composite walls. The parametric values considered in the analysis are shown in Table 6 and are all nominal ones, representing low, medium and high levels of each parameter in practical engineering. Three analysis cases were assigned to each parameter. For the 
axial load ratio, the three values $0,0.13$ and 0.26 were considered and represented by case N1, N2 and N3, respectively. For the steel ratio of embedded truss chord, the three values of $1.73,3.59$ and $5.38 \%$ were considered and represented by $\mathrm{C} 1, \mathrm{C} 2$ and C3, respectively. For the volumetric steel ratio of embedded truss web braces, the three values $0.70,1.59$ and $2.36 \%$ were considered and represented by B1, B2 and B3, respectively. Therefore, in order to identify a numerical model for the parametric analysis, three parameters need to be specified. For example, the numerical model S-N1-C1-B1 denotes the model with zero axial load ratio, $1.73 \%$ of steel ratio of embedded truss chord, and $0.70 \%$ of volumetric ratio of embedded truss web braces. Each numerical model was subjected to the same loading history as the specimen in the experimental program. The computed lateral load versus lateral displacement envelope curves were plotted and compared in Figs. 14-16, along with the results summarized in Tables 7-15.

Table 6 Range of parametric analysis

\begin{tabular}{|c|c|c|c|c|c|c|c|}
\hline \multicolumn{2}{|c|}{$\alpha$} & \multicolumn{2}{c|}{$\rho_{\mathrm{c}}$} & \multicolumn{3}{c|}{$\rho_{\mathrm{vb}}$} \\
\hline Case & Value & Case & Type & $\rho_{\mathrm{c}}(\%)$ & Case & Type & $\rho_{\mathrm{vb}}(\%)$ \\
\hline N1 & 0.0 & C1 & C5 & 1.73 & B1 & $2\llcorner 25 \times 2$ & 0.70 \\
\hline N2 & 0.13 & C2 & I10 & 3.59 & B2 & $2\llcorner 30 \times 4$ & 1.59 \\
\hline N3 & 0.26 & C3 & I14 & 5.38 & B3 & $2\llcorner 36 \times 5$ & 2.36 \\
\hline
\end{tabular}

\subsection{Effect of axial load ratio $\alpha$}

The influence of axial load ratio $\alpha$ on the lateral load-displacement skeleton curves is shown in Fig. 14(a)-(i) for various combinations of steel ratio of embedded truss chord $\rho_{\mathrm{c}}$ and volumetric ratio of embedded truss web braces $\rho_{\mathrm{vb}}$. As can be seen, for a certain combination of $\rho_{\mathrm{c}}$ and $\rho_{\mathrm{vb}}$, much higher initial stiffness and lateral load 
capacity can be obtained under higher axial load ratio. Table 7 further summarizes the influence of $\alpha$ on the lateral load capacity, indicating approximately an average of $26 \%$ increase when $\alpha$ is increased from zero to 0.26 . Table 8 shows the effect of $\alpha$ on the energy dissipation capacity. The increase of axial load ratio will cause the significant decrease of total dissipated energy before failure. The displacement ductility coefficient of each case is also calculated and summarized in Table 9. When the axial load ratio is increased from zero to 0.13 , the corresponding displacement ductility coefficient is also increased by up to $50 \%$ and an average of $22 \%$. However, when the axial load ratio is further increased to 0.26 , the resulting displacement ductility coefficient is decreased by up to $30 \%$ and an average of $23 \%$ as compared with the medium level axial load ratio.

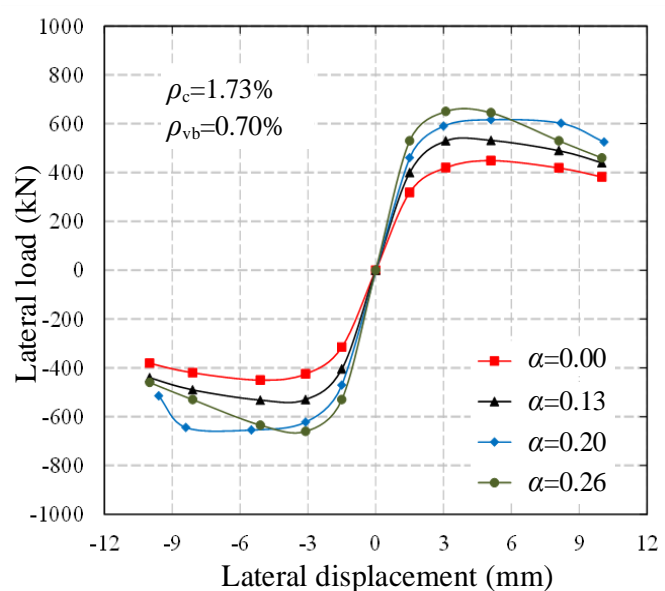

(a) S-N-C1-B1

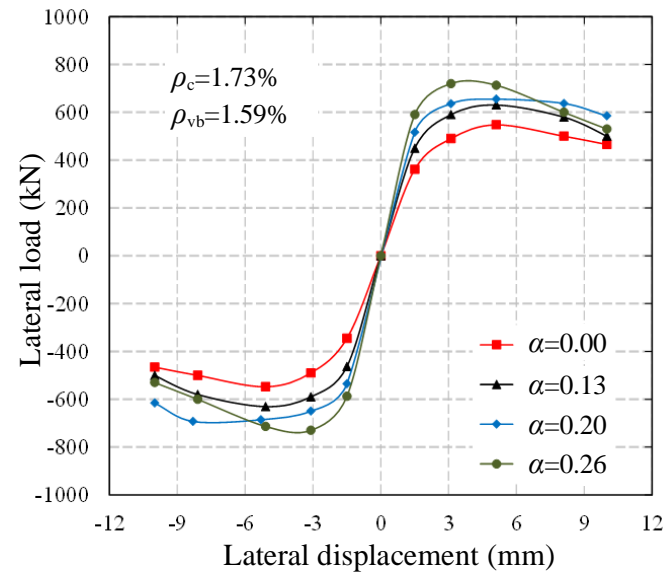

(b) S-N-C1-B2 


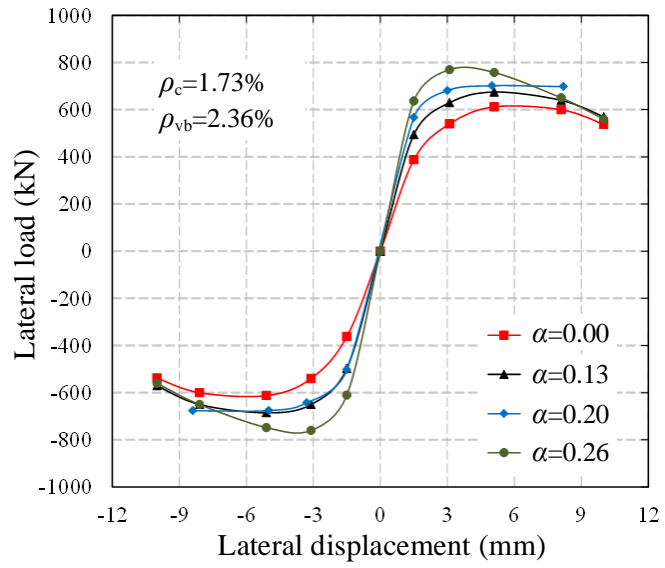

(c) S-N-C1-B3

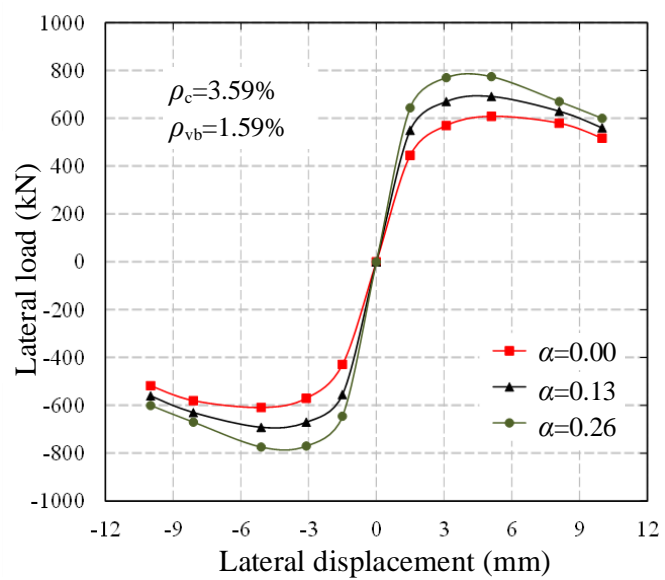

(e) S-N-C2-B2

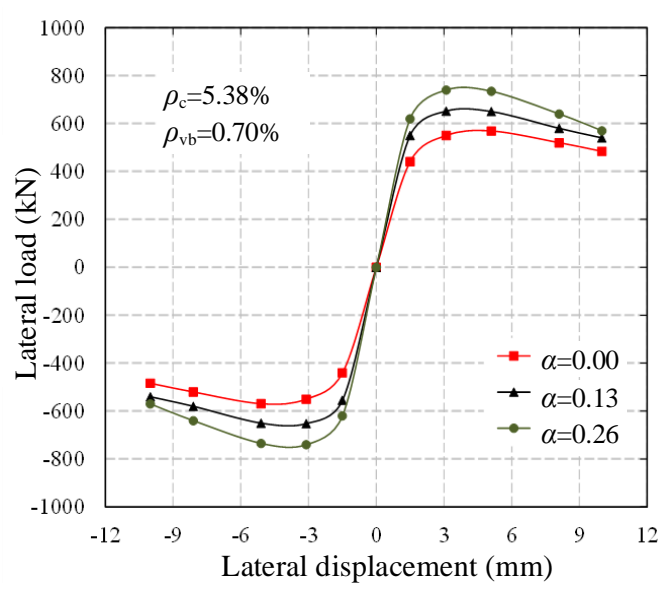

(g) S-N-C3-B1

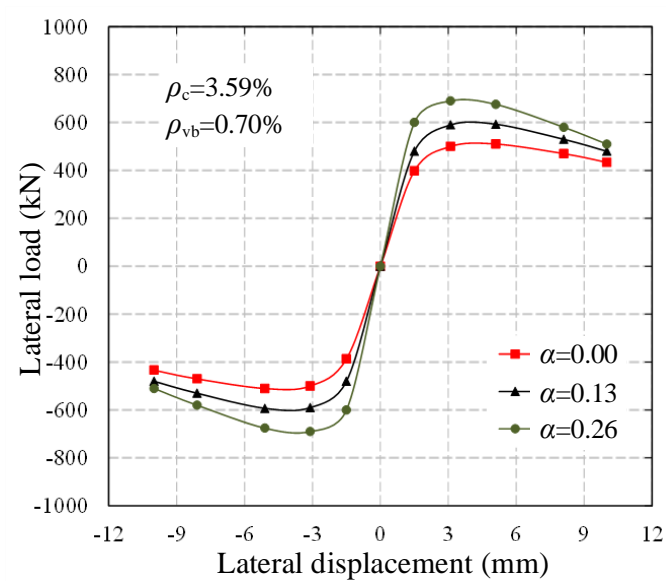

(d) S-N-C2-B1

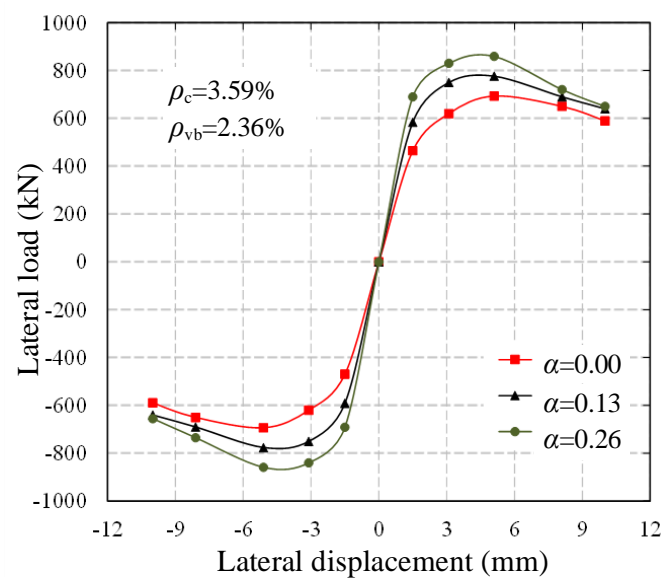

(f) $\mathrm{S}-\mathrm{N}-\mathrm{C} 2-\mathrm{B} 3$

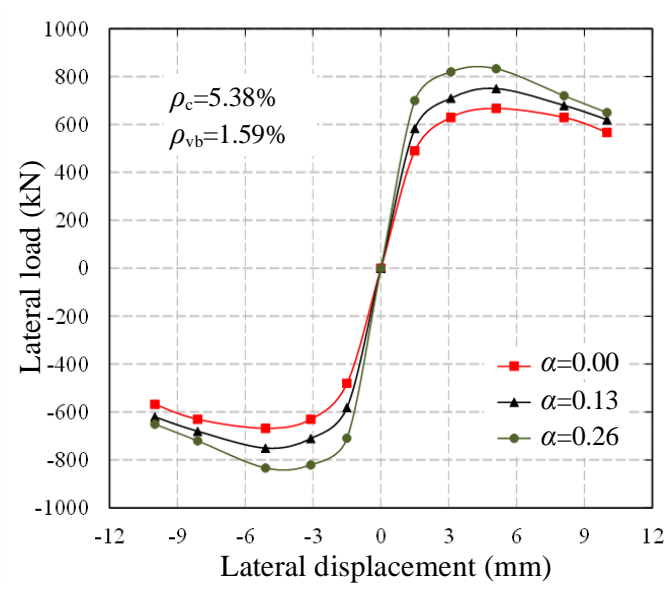

(h) S-N-C3-B2 


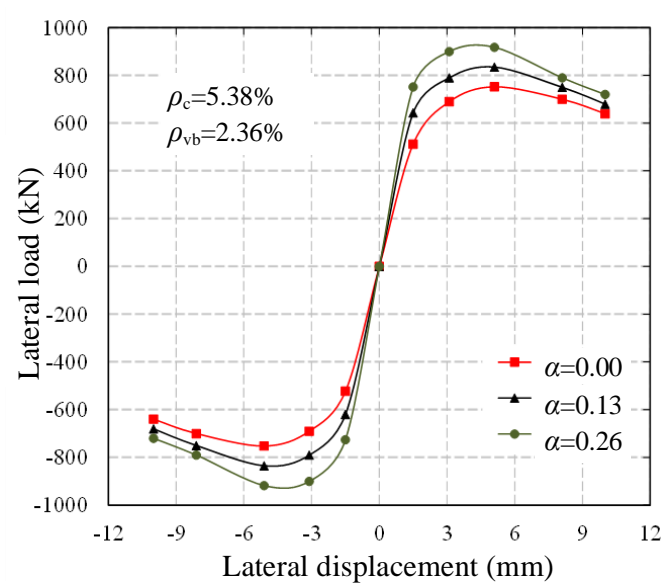

(i) $\mathrm{S}-\mathrm{N}-\mathrm{C} 3-\mathrm{B} 3$

Fig. 14 Effect of axial load ratio, $\alpha$

Table 7 Effect of $\alpha$ on lateral load capacity (kN)

\begin{tabular}{|c|c|c|c|c|}
\hline & & $\alpha=0.00$ & $\alpha=0.13$ & $\alpha=0.26$ \\
\hline \multirow{3}{*}{$\rho_{\mathrm{c}}=1.73 \%$} & $\rho_{\mathrm{vb}}=0.70 \%$ & 449.5 & 532.1 & 655.0 \\
\hline & $\rho_{\mathrm{vb}}=1.59 \%$ & 547.8 & 630.5 & 725.0 \\
\hline & $\rho_{\mathrm{vb}}=2.36 \%$ & 612.3 & 680.1 & 765.0 \\
\hline \multirow{3}{*}{$\rho_{\mathrm{c}}=3.59 \%$} & $\rho_{\mathrm{vb}}=0.70 \%$ & 510.3 & 593.0 & 690.0 \\
\hline & $\rho_{\mathrm{vb}}=1.59 \%$ & 608.7 & 691.4 & 774.2 \\
\hline & $\rho_{\mathrm{vb}}=2.36 \%$ & 693.2 & 776.0 & 858.8 \\
\hline \multirow{3}{*}{$\rho_{\mathrm{c}}=5.38 \%$} & $\rho_{\mathrm{vb}}=0.70 \%$ & 569.1 & 651.9 & 740.0 \\
\hline & $\rho_{\mathrm{vb}}=1.59 \%$ & 667.5 & 750.3 & 833.0 \\
\hline & $\rho_{\mathrm{vb}}=2.36 \%$ & 752.1 & 834.8 & 917.6 \\
\hline
\end{tabular}

Table 8 Effect of $\alpha$ on energy dissipation capacity $(\mathrm{N} \cdot \mathrm{mm})$

\begin{tabular}{|c|c|c|c|c|}
\hline \multicolumn{2}{|c|}{} & $\alpha=0.00$ & $\alpha=0.13$ & $\alpha=0.26$ \\
\hline \multirow{3}{*}{$\rho_{\mathrm{c}}=1.73 \%$} & $\rho_{\mathrm{vb}}=0.70 \%$ & 26465.0 & 19703.7 & 14297.2 \\
\cline { 2 - 5 } & $\rho_{\mathrm{vb}}=1.59 \%$ & 31329.4 & 23626.2 & 17471.6 \\
\cline { 2 - 5 } & $\rho_{\mathrm{vb}}=2.36 \%$ & 32341.4 & 25394.8 & 19116.5 \\
\hline \multirow{3}{*}{$\rho_{\mathrm{c}=3.59 \%}$} & $\rho_{\mathrm{vb}}=0.70 \%$ & 27176.2 & 20142.0 & 14624.5 \\
\cline { 2 - 5 } & $\rho_{\mathrm{vb}}=1.59 \%$ & 32959.0 & 23502.9 & 17416.3 \\
\cline { 2 - 5 } & $\rho_{\mathrm{vb}}=2.36 \%$ & 34170.9 & 25683.5 & 18760.5 \\
\hline \multirow{3}{*}{$\rho_{\mathrm{c}}=5.38 \%$} & $\rho_{\mathrm{vb}}=0.70 \%$ & 27089.0 & 18479.1 & 14543.9 \\
\cline { 2 - 5 } & $\rho_{\mathrm{vb}}=1.59 \%$ & 34006.6 & 23543.3 & 17150.9 \\
\cline { 2 - 5 } & $\rho_{\mathrm{vb}}=2.36 \%$ & 34772.6 & 25146.2 & 18372.6 \\
\hline
\end{tabular}

Table 9 Effect of $\alpha$ on displacement ductility

\begin{tabular}{|c|c|c|c|c|}
\hline \multicolumn{2}{|c|}{} & $\alpha=0.00$ & $\alpha=0.13$ & $\alpha=0.26$ \\
\hline \multirow{3}{*}{$\rho_{\mathrm{c}}=1.73 \%$} & $\rho_{\mathrm{vb}}=0.70 \%$ & 4.31 & 5.62 & 4.15 \\
\cline { 2 - 5 } & $\rho_{\mathrm{vb}}=1.59 \%$ & 5.27 & 6.42 & 5.06 \\
\cline { 2 - 5 } & $\rho_{\mathrm{vb}}=2.36 \%$ & 6.05 & 6.87 & 5.73 \\
\hline
\end{tabular}




\begin{tabular}{|c|c|c|c|c|}
\hline \multirow{3}{*}{$\rho_{\mathrm{c}}=3.59 \%$} & $\rho_{\mathrm{vb}}=0.70 \%$ & 5.09 & 5.84 & 4.82 \\
\cline { 2 - 5 } & $\rho_{\mathrm{vb}}=1.59 \%$ & 6.22 & 7.07 & 6.35 \\
\cline { 2 - 5 } & $\rho_{\mathrm{vb}}=2.36 \%$ & 6.86 & 7.89 & 6.92 \\
\hline \multirow{3}{*}{$\rho_{\mathrm{c}}=5.38 \%$} & $\rho_{\mathrm{vb}}=0.70 \%$ & 5.73 & 6.97 & 5.93 \\
\cline { 2 - 5 } & $\rho_{\mathrm{vb}}=1.59 \%$ & 6.49 & 7.62 & 6.65 \\
\cline { 2 - 5 } & $\rho_{\mathrm{vb}}=2.36 \%$ & 7.34 & 8.24 & 7.21 \\
\hline
\end{tabular}

\subsection{Effect of steel ratio $\rho_{c}$ of embedded truss chord}

The effect of steel ratio $\rho_{\mathrm{c}}$ of the embedded truss chord on the backbone curves between the lateral load and lateral displacement is shown in Fig. 15(a)-(i) for different combinations of $\alpha$ and $\rho_{\mathrm{vb}}$. Three steel ratios $\rho_{\mathrm{c}}$ of $1.73,3.59$ and $5.38 \%$ were considered. The higher $\rho_{\mathrm{c}}$ value can result in higher initial stiffness, while the influence of $\rho_{\mathrm{c}}$ on the initial stiffness becomes more and more insignificant as the $\alpha$ value increases. It can be seen that higher $\rho_{\mathrm{c}}$ can lead to larger lateral load capacity. Table 7 summarizes the influence of $\rho_{\mathrm{c}}$ on the lateral load capacity. There is an average of $19 \%$ increase in the lateral load capacity when $\rho_{\mathrm{c}}$ is increased from 1.73 to $5.38 \%$. Table 8 shows the insignificant influence of $\rho_{\mathrm{c}}$ on the energy dissipation capacity. It turns out that increasing $\rho_{\mathrm{c}}$ does not necessarily increase the energy dissipation capacity. Table 9 summarizes the influence of $\rho_{\mathrm{c}}$ on the displacement ductility. Under a low to medium axial load ratio, increasing $\rho_{\mathrm{c}}$ can slightly improve the ductility behavior. However, higher $\rho_{\mathrm{c}}$ has little influence on the displacement ductility coefficient when subjected to high axial load ratio of 0.26 . 


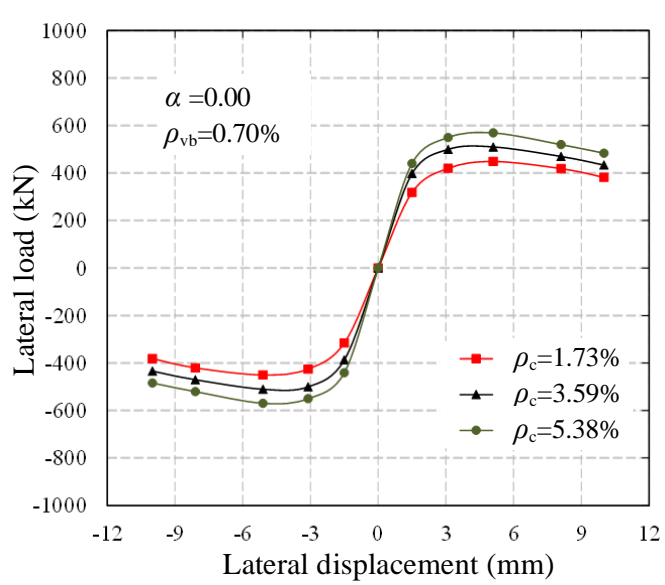

(a) S-N1-C1-B

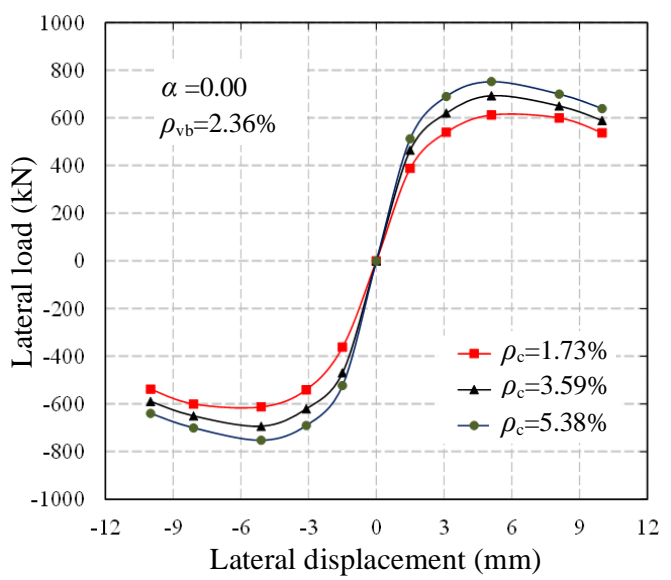

(c) S-N1-C3-B

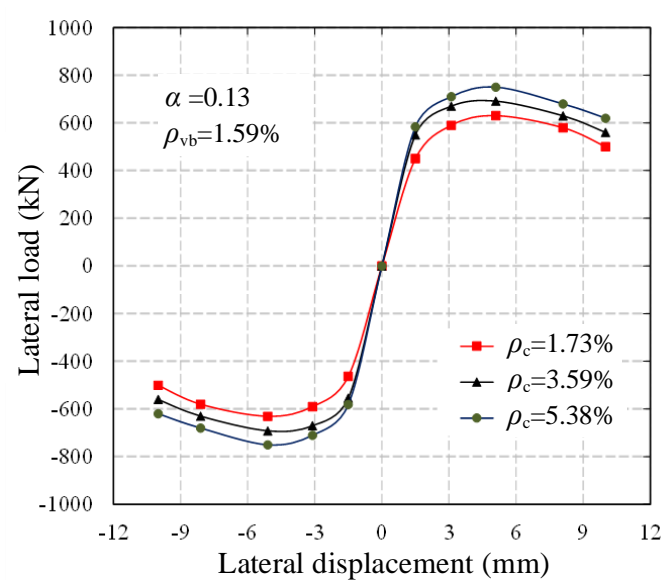

(e) S-N2-C2-B

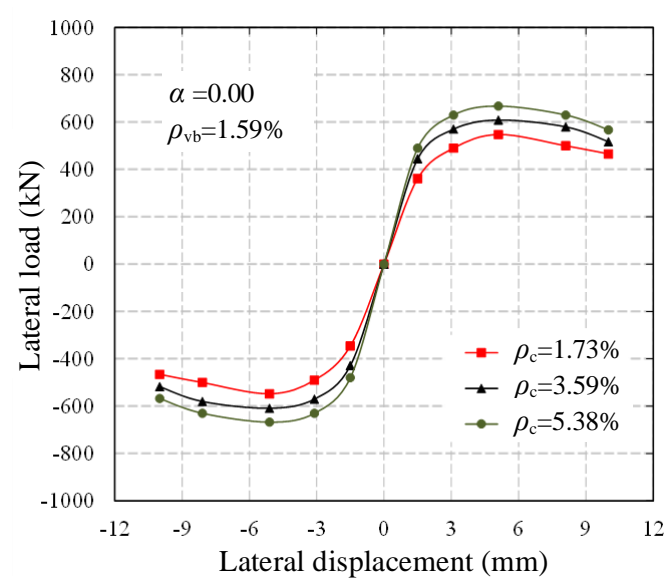

(b) S-N1-C2-B

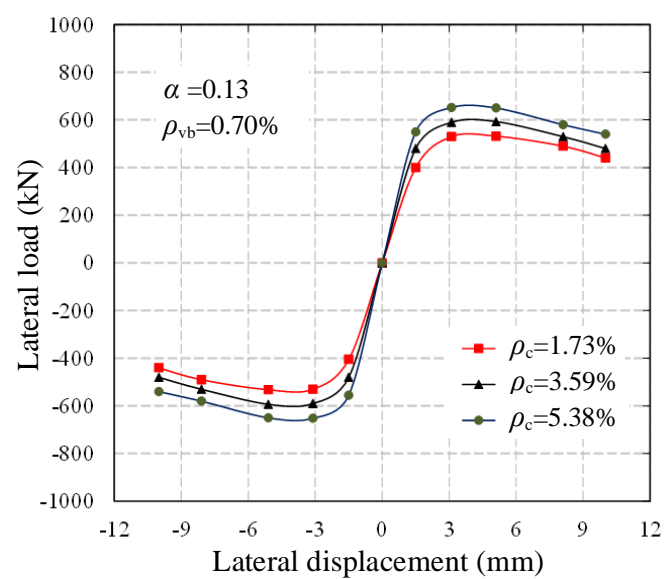

(d) S-N2-C1-B

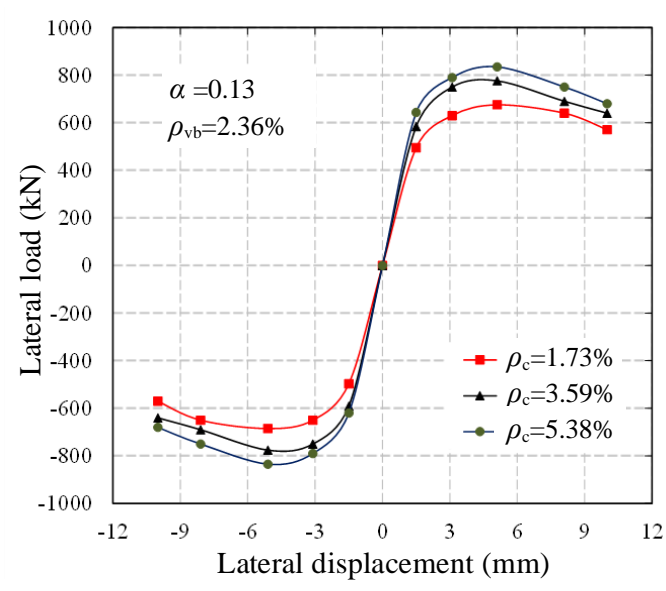

(f) S-N2-C3-B 


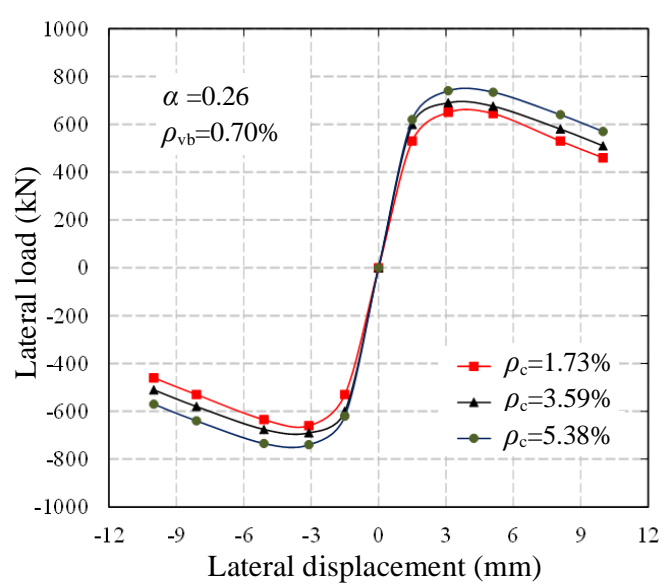

(g) S-N3-C1-B

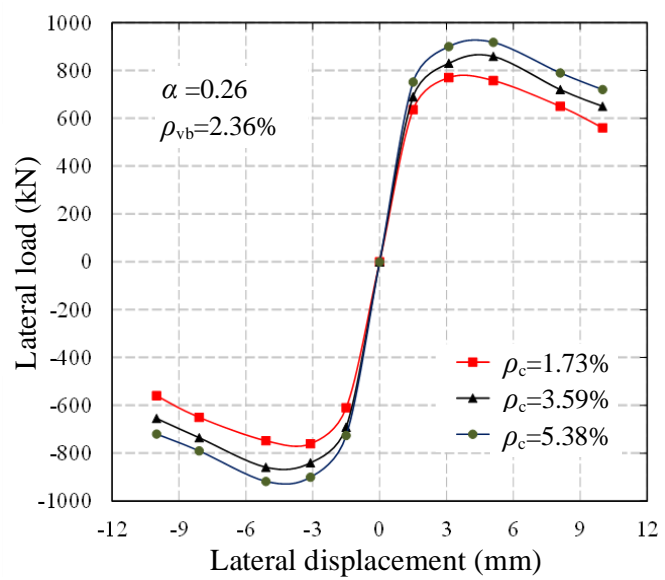

(i) S-N3-C3-B

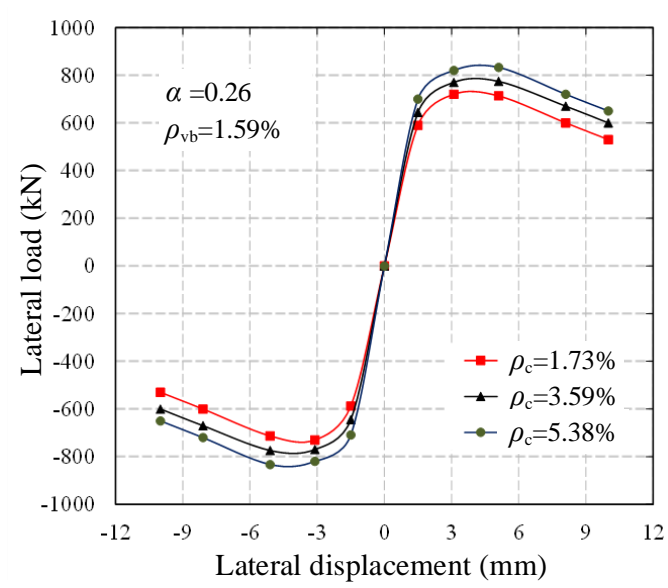

(h) S-N3-C2-B

Fig. 15 Influence of steel ratio $\rho_{\mathrm{c}}$ of embedded truss chord

\subsection{Effect of volumetric ratio of embedded truss web braces $\rho_{v b}$}

The effect of volumetric ratio $\rho_{\mathrm{vb}}$ of the embedded truss web brace on the backbone curve of the composite walls is shown in Fig. 16(a)-(i) for three values of $\rho_{\mathrm{vb}}, 0.7$, 1.59 and $2.36 \%$. The specimens with different $\rho_{\mathrm{vb}}$ behave almost identical prior to yielding, but the post-yielding behavior varies. Under certain combination of $\alpha$ and $\rho_{\mathrm{c}}$, higher $\rho_{\mathrm{vb}}$ can effectively increase the lateral load capacity by up to $37 \%$ and an average of $29 \%$. Table 7 summarizes the lateral load capacity with different combinations of $\alpha, \rho_{\mathrm{c}}$ and $\rho_{\mathrm{vb}}$. Table 8 shows the influence of $\rho_{\mathrm{vb}}$ on the energy dissipation capacity. Clearly, by increasing $\rho_{\mathrm{vb}}$ from 0.7 to $1.59 \%$, the energy 
dissipation capacity of the specimens can be increased by up to $21 \%$. However, increasing $\rho_{\mathrm{vb}}$ from 1.59 to $2.36 \%$, the energy dissipation capacity can be increased by only $6 \%$. Thus a medium level amount of embedded truss web brace is most efficient in terms of improving the energy dissipation capacity of specimens. Table 9 shows the influence of $\rho_{\mathrm{vb}}$ on displacement ductility. Similar pattern to energy dissipation capacity can be found in that when medium level amount of embedded truss web brace is used the displacement ductility coefficient can be most effectively improved as compared with a high level of $\rho_{\mathrm{vb}}$.

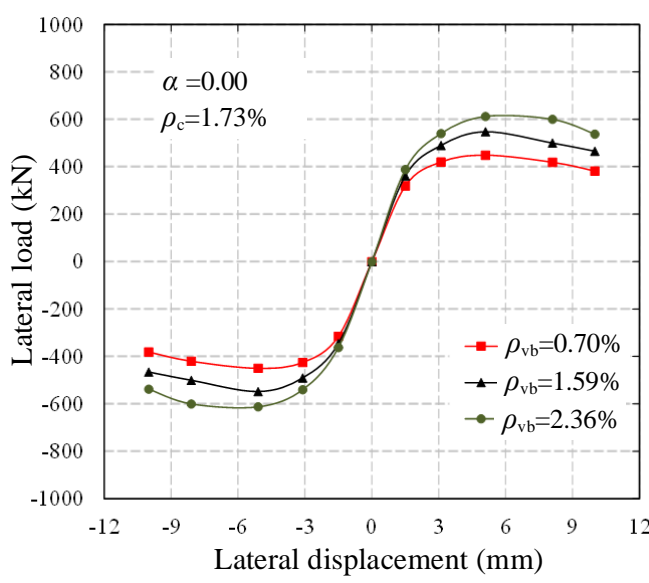

(a) S-N1-C-B1

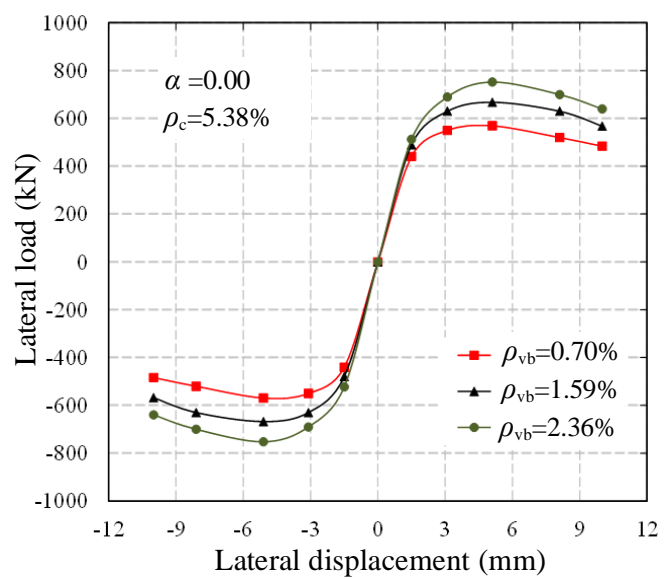

(c) S-N1-C-B3

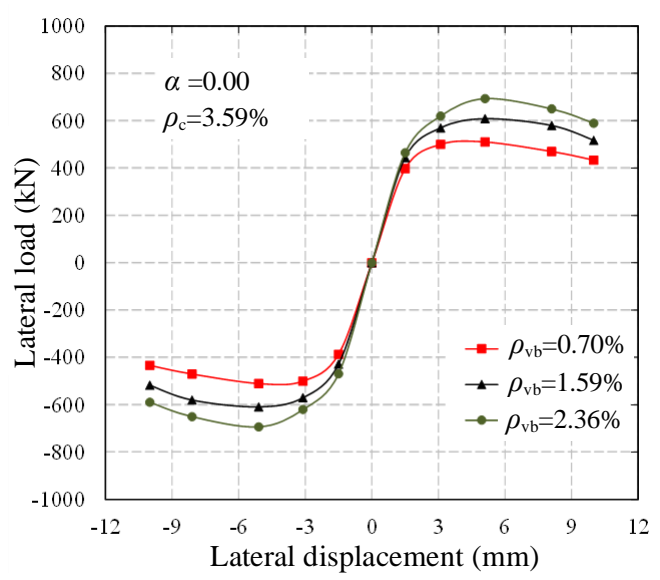

(b) S-N1-C-B2

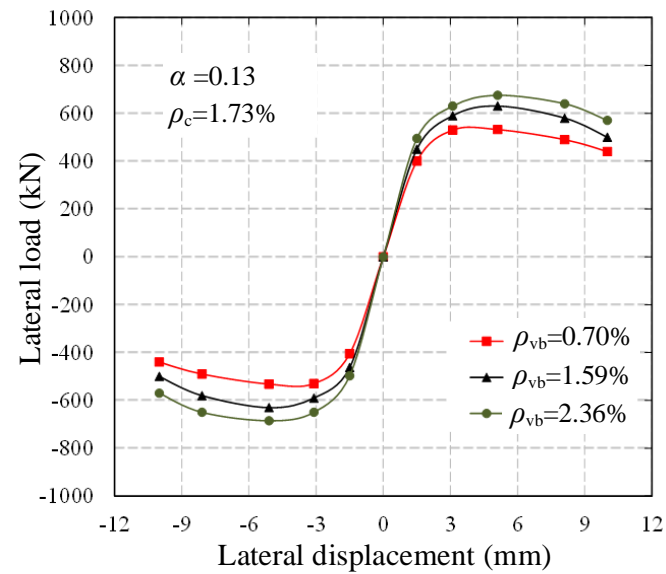

(d) S-N2-C-B1 


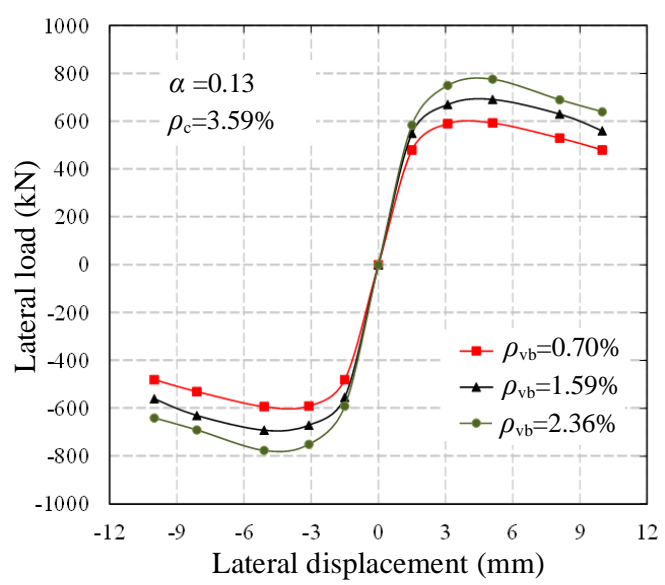

(e) S-N2-C-B2

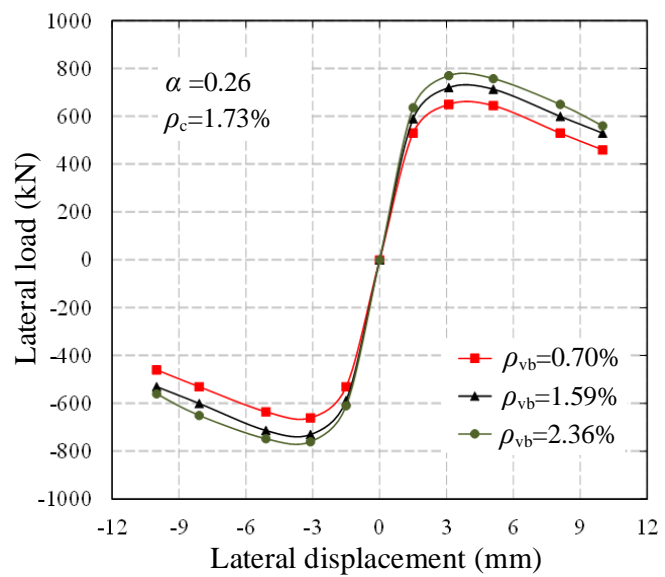

(g) S-N3-C-B1

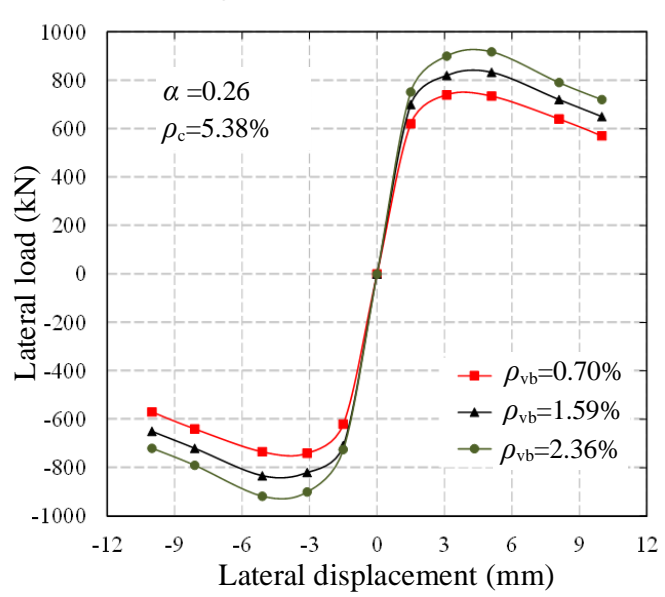

(i) S-N3-C-B3

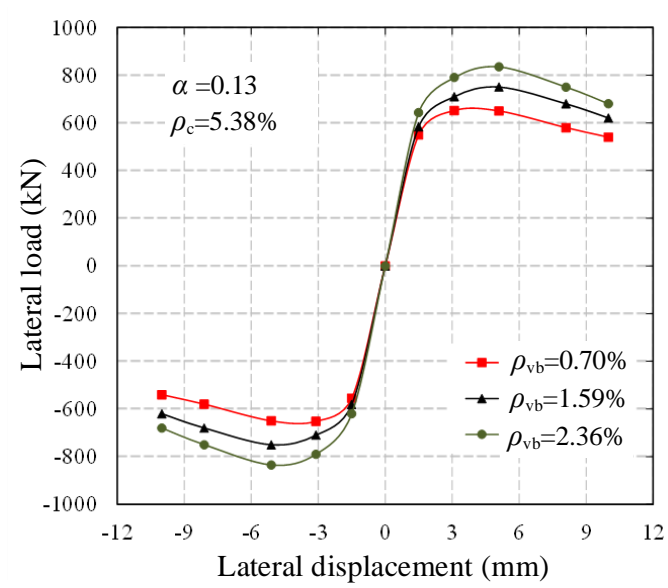

(f) S-N2-C-B3

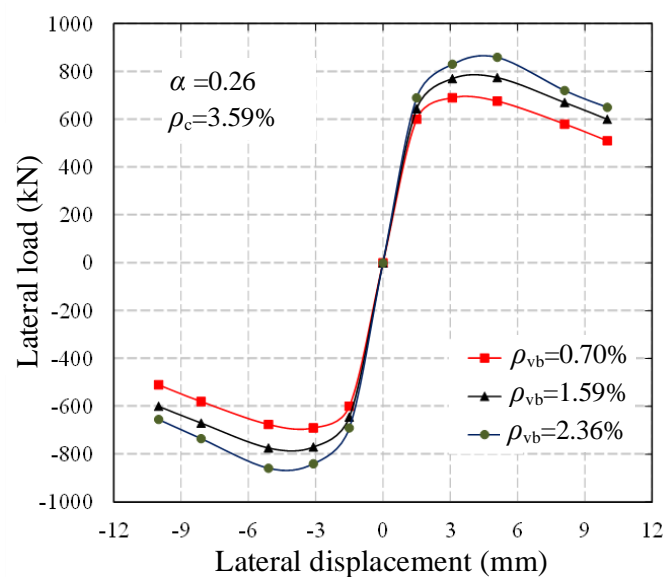

(h) S-N3-C-B2

Fig. 16 Influence of volumetric ratio of embedded truss web brace, $\rho_{\mathrm{vb}}$

\section{Conclusion}

The seismic performance of steel and concrete composite shear walls with an aspect ratio of 1.0 embedded with steel truss was investigated by both an experimental 
program and a parametric analysis, revealing the different contributions of the embedded truss chord and web brace to the overall seismic performance of the composite walls. Based on the experimental and numerical analysis, the following conclusions can be made:

(1) The experimental results show that the embedded truss web brace can improve the hysteretic behavior of composite walls in terms of lateral load capacity, energy dissipation capacity and ductility. The embedded truss chord, on the other hand, can enhance the lateral load capacity of the composite walls.

(2) Good agreement has been achieved between the experimental and simulation results. This indicates that the present finite element modeling technique can be reliably used to analyze the overall seismic behavior of the composite walls embedded with steel truss. The summation of the strength contributions by RC wall and the embedded steel truss can provide a good estimate of the resistance of the composite system.

(3) High axial load ratios and high steel ratios of embedded truss chord are beneficial to initial stiffness and lateral load capacity. However, high axial load ratios are adverse to energy dissipation capacity.

(4) The influence of steel ratio of embedded truss chord on the energy dissipation capacity and ductility is insignificant for composite walls with low aspect ratios, since the lateral load and post-yield deformation capacity are mainly provided by the web region. 
(5) For composite walls with low aspect ratios, the volumetric ratio of embedded truss web brace is most effective in increasing the lateral load capacity, while has little influence on the initial stiffness.

(6) From the perspective of seismic design, optimum design is suggested according to the parametric analysis results. Particularly, medium level of volumetric ratio about $1.59 \%$ of embedded truss web brace is most efficient in ensuring satisfactory energy dissipation capacity and ductility as compared with the other two parameters. In order to avoid the adverse effect on ductility behavior, the axial load ratio should be limited to the medium level, about 0.13 for the nominal value or about 0.30 for the design value.

\section{Nomenclature}

$V_{n}$ : the ideal lateral load capacity of the composite wall

$V_{\mathrm{RC}}$ : the shear capacity of concrete encasement

$V_{\text {st: }}$ the shear capacity of steel truss

$u$ : the displacement ductility coefficient

$\Delta_{\mathrm{u}}$ : the corresponding horizontal displacement when the tests were terminated

$\Delta_{\mathrm{y}}$ : the lateral displacement at yield

$h_{\mathrm{w}}$ : the height of shear wall

$l_{\mathrm{w}}$ : the width of shear wall

$\alpha$ : the axial load ratio.

$\rho_{\mathrm{c}}$ : the steel ratio of the embedded truss chord

$\rho_{\mathrm{vb}}$ : the volumetric ratio of the embedded truss web braces 
$\varepsilon_{\mathrm{c} / 3}$ : the strain at which one-third of the maximum compressive strength is reached $\varepsilon c$ : the strain at which the maximum compressive strength is reached $\varepsilon u$ : the ultimate strain at which the material is completely softened in compression $f_{c}$ : compressive strength of concrete

$f_{\mathrm{t}}$ : tensile strength of concrete

$E$ : Young's modulus

$G_{\mathrm{c}}:$ the total compressive fracture energy

$G_{\mathrm{F}}:$ the total tensile fracture energy

$h$ : the characteristic element length

$\tau_{\max }$ : the bond strength

$\tau_{\mathrm{f}}:$ the residual bond stress

$s:$ the relative slip between steel and concrete

$\beta$ : the empirical coefficient.

\section{Acknowledgments}

This research project is financially sponsored by the National Science Foundation of China under the grant number of 51578090 and the grant by Chongqing Science and Technology Commission under the contract number of CSTC2015JCYJYS0004. The authors would like to express their sincere thanks and appreciation to various supporting agencies of this project.

\section{References}

[1] Zhao QH, Abolhassan AA, Cyclic behavior of traditional and innovative composite shear wall, J. Struct. Eng. 130(2) (2004) 271-84. 
[2] Dan D, Fabian A and Stoian V, Theoretical and experimental study on composite steel-concrete shear wall with vertical steel encased profiles, J. Constr. Steel Res. 67(5SI) (2011) 800-13.

[3] Qian JR, Jiang Z and Ji XD, Behavior of steel tube-reinforced concrete composite walls subjected to high axial force and cyclic loading, Eng. Struct. 36 (2012) 173-84.

[4] Nie JG, Hu HS, Fan JS, Tao M, Li SY and Liu FJ, Experimental study on seismic behavior of high-strength concrete filled double-steel-plate composite walls, J. Constr. Steel Res. 88 (2013) 206-19.

[5] Rafiei S, Hossain KMA, Lachemi M, and Behdinan K, Composite wall with high performance concrete subjected to monotonic shear, J. Constr. Steel Res. 107 (2015) 124-36.

[6] Hossain KMA, Rafiei S, Lachemi M, Behdinan K, Structural performance of profiled composite wall under in-plane cyclic loading, Eng. Struct. 110 (2016) 88104.

[7] Chen L, Mahmoud H, Tong SM, Zhou Y, Seismic behavior of double steel plate-HSC composite walls, Eng. Struct. 102 (2015) 1-12.

[8] Sittipunt C, Wood SL, and Lukkunaprasit P, Cyclic behavior of reinforced concrete shear walls with diagonal web reinforcement, ACI Structural Journal, 98(4) (2001) 554-562.

[9] Shaingchin S, Lukkunaprasit P and Wood SL, Influence of diagonal web reinforcement on cyclic behavior of structural walls, Eng. Struct. 29 (2007) 498-510. [10] (GB50011-2010) Code for seismic design of buildings. (in Chinese) 
[11] Park, R., Ductility evaluation from laboratory and analytical testing, Proceedings of the 9th World Conference on Earthquake Engineering, 8(1988), 605-616, Tokyo-Kyoto, Japan.

[12] DIANA 9.4.3, Delft, The Netherlands (2012), TNO DIANA BV.

[13] Feenstra PH, Computational Aspects of Biaxial Stress in Plain and Reinforced Concrete, PhD Thesis, Delft University of Technology (1993).

[14] (GB50010-2010) Code for design of concrete structures. (in Chinese)

[15] Telford T. CEB-FIP Model Code-1990. CEB Bulletin No. 213/214, Lausanne, Switzerland (1993). 